\title{
Poroma écrino associado a um hidradenoma poroide devido à coexistência de uma grande massa sobre o pé: Relato de caso*
}

\section{A Large Mass over the Foot due to the Coexistence of an Eccrine Poroma and a Poroid Hidradenoma: A Case Report}

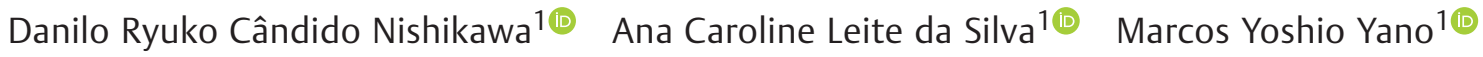 \\ Bruno Rodrigues de Miranda1이 Wu Tu Chung ${ }^{1}{ }^{\circ}$
}

${ }^{1}$ Departamento de Ortopedia, Cirurgia do Pé e Tornozelo, Hospital do Servidor Público Municipal de São Paulo, São Paulo, SP, Brasil

Rev Bras Ortop
Endereço para correspondência Danilo Ryuko Cândido Nishikawa, MD, Department of Orthopaedic, Foot and Ankle Surgery, Hospital do Servidor Público Municipal de São Paulo, Rua Castro Alves, 60, Aclimação, São Paulo, SP, 01532-000, Brasil (e-mail: dryuko@gmail.com).

\section{Resumo \\ O poroma écrino e o hidradenoma poroide são neoplasias poroides benignas raras, derivadas das glândulas sudoríparas écrinas. Existem quatro tipos de neoplasias poroides, conforme a posição na camada da pele, sendo denominadas hidroacantoma simples, poroma écrino, tumor do ducto dérmico e hidradenoma poroide. As neo- plasias poroides geralmente surgem como uma lesão solitária, com crescimento lento, podendo apresentar diferentes apresentações clínicas, como massa sobre o pé, lesão ulcerada, cisto sólido, lesão hemorrágica ou suspeita de melanoma. As extremidades são os sítios mais comuns, especialmente as mãos e os pés. No entanto, a coexistência desses dois tumores em uma única lesão é extremamente rara. A excisão cirúrgica representa o principal tratamento, podendo ser curativa, prevenindo assim as altera- \\ Palavras-chave \\ - pé \\ - tumor \\ - poroma écrino \\ - hidradenoma poroide ções malignas e as recidivas. Descrevemos um raro tumor solitário no pé com características clínicas e histopatológicas de uma associação de poroma écrino e hidradenoma poroide tratado cirurgicamente sem recidiva no acompanhamento de médio prazo. \\ Nível de Evidência IV, Relato de Caso.}

Trabalho desenvolvido no Serviço de Ortopedia, Cirurgia do Pé e Tornozelo do Hospital do Servidor Público Municipal de São Paulo, São Paulo, SP, Brasil recebido

03 de Abril de 2021

aceito

23 de Abril de 2021
DOI https://doi.org/

10.1055/s-0041-1732331. ISSN 0102-3616. (c) 2021. Sociedade Brasileira de Ortopedia e Traumatologia. All rights reserved.

This is an open access article published by Thieme under the terms of the Creative Commons Attribution-NonDerivative-NonCommercial-License, permitting copying and reproduction so long as the original work is given appropriate credit. Contents may not be used for commercial purposes, or adapted, remixed, transformed or built upon. (https://creativecommons.org/ licenses/by-nc-nd/4.0/)

Thieme Revinter Publicações Ltda., Rua do Matoso 170, Rio de Janeiro, RJ, CEP 20270-135, Brazil 


\begin{abstract}

\section{Keywords}

- foot

- tumor

- eccrine poroma

- poroid hidradenoma

Eccrine poroma and poroid hidradenoma are uncommon benign poroid neoplasms derived from eccrine sweat glands. There are four types of poroid neoplasms according to the position within the skin layer: hidroacanthoma simplex, eccrine poroma, dermal duct tumor, and poroid hidradenoma. Poroid neoplasms usually arise as slow-growing solitary lesions and can present different clinical presentations, such as a foot mass, an ulceration lesion, a solid cyst, a bleeding lesion or suspected melanoma. Extremities are the most common sites, especially hands and feet. However, the coexistence of these two tumors in a single lesion is extremely rare. Surgical excision represents the main treatment and can be curative, preventing malignant changes and recurrence. We describe a rare solitary tumor over the foot with clinical and histopathological features of an association of an eccrine poroma and a poroid hidradenoma that was surgically treated with no recurrence at the midterm follow-up.

Level of Evidence IV, Case Report.
\end{abstract}

\section{Introdução}

O poroma écrino (PE) e o hidradenoma poroide (HP) são neoplasias poroides (NPs) benignas raras derivadas das glândulas sudoríparas écrinas. ${ }^{1-3}$ As NPs geralmente surgem como uma lesão solitária com diferentes manifestações clínicas, como massa sobre o pé, lesão ulcerada, cisto sólido, lesão hemorrágica ou suspeita de melanoma. Existem quatro tipos de variações de NP, classificadas em relação à posição dentro da camada da pele: hidroacantoma simples, PE, tumor do ducto dérmico e HP. ${ }^{4} \mathrm{O}$ PE envolve a camada basal da epiderme, enquanto o HP está confinado à derme..$^{3-5}$

A literatura sobre a coexistência desses dois tumores em uma única lesão é escassa. ${ }^{5-7}$ A ressecção total seguida de biópsia tem sido descrita como o tratamento principal, e pode ser curativa. ${ }^{2} \mathrm{O}$ presente estudo tem como objetivo relatar um caso com uma grande massa solitária sobre o pé, originado a partir de uma rara associação de PE e HP.

\section{Apresentação do Caso}

Apresentamos um paciente do sexo masculino de 55 anos com uma grande massa não traumática e dolorosa sobre o médio-pé esquerdo $(\sim 7 \times 4 \mathrm{~cm})(-$ Fig. 1$)$. Ele referiu que a lesão começou como uma mancha nodular hipercrômica na pele e cresceu lentamente nos últimos 8 anos. Ao exame físico, o tumor apresentava bordas bem definidas e consistência fibroelástica, não aderindo ao tecido mole circundante. A mancha nodular hipercrômica tinha o tamanho aproximado de $1,5 \times 1 \mathrm{~cm}$.

Foi realizada a ultrassonografia para confirmação de sua forma cística e análise do conteúdo. 0 resultado foi uma formação cística translúcida bem definida medindo $72 \times 46$ $\times 26 \mathrm{~mm}$, com $3 \mathrm{~mm}$ de um conteúdo fluido homogêneo dentro do tecido subcutâneo (-Fig. 2). Havia a suspeita de que o tumor cístico poderia ser um cisto sinovial comum. Porém, solicitamos avaliação dermatológica, devido à presença de uma mancha preta na pele sobre o cisto. Cogitou-se a hipótese de que o diagnóstico diferencial seria melanoma, carcinoma basocelular pigmentado e neoplasias epiteliais benignas.

Foi realizada a ressecção total do tumor, já que o seu aspecto clínico e as imagens ultrassonográficas apresentavam características benignas. Foi realizada uma abordagem dorsomedial longitudinal centralizada sobre o tumor a partir da articulação do tornozelo até o terço distal do primeiro metatarso, sendo a mancha preta removida totalmente em forma de elipse. Nossa decisão por esta abordagem foi porque ela permitiria dissecar o tumor medial e lateralmente, com menos manipulação dos tecidos moles, já que a pele sobre os tumores císticos tende a ser fina (-Fig. 3). Durante a dissecção, foi tomado todo o cuidado para evitar danos ao ramo nervoso do nervo fibular profundo, ao nervo safeno e à veia, bem como à artéria dorsal do pé. Conseguimos dissecar e excisar totalmente o tumor com uma margem limpa (-Fig. 3). Em seguida, o tumor foi encaminhado para análise anatomopatológica.

O exame histopatológico confirmou que o tumor era uma $\mathrm{NP}$, consistindo de ambos os componentes da derme

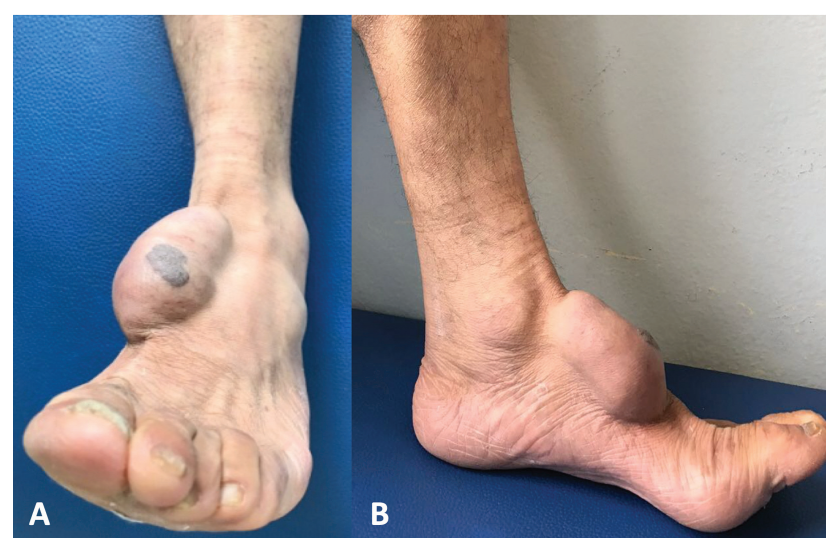

Fig. 1 (A) Vista clínica frontal e (B) lateral de grande massa sobre o médio-pé, com bordas bem definidas, consistência fibroelástica e não aderente ao tecido mole circundante. O nódulo preto ligeiramente elevado na parte superior é a porção PE, e a maior parte do tumor cístico é a porção HP. 


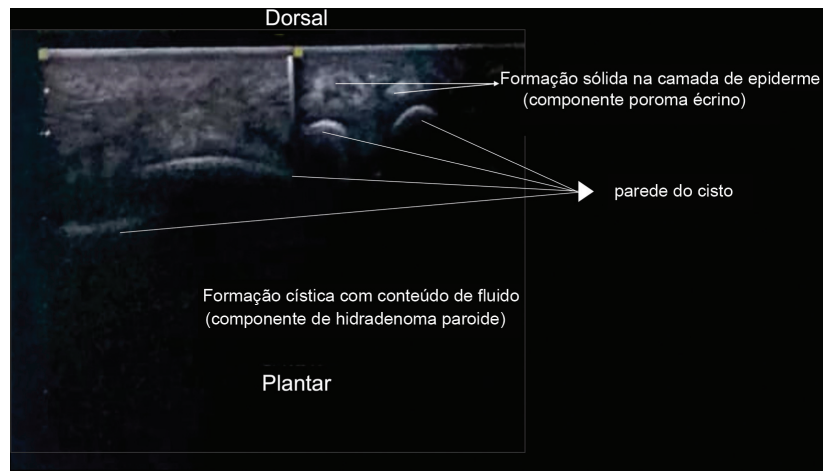

Fig. 2 Imagem de ultrassom mostrando uma formação cística translúcida bem definida, apresentando um conteúdo fluido homogêneo dentro da camada derme (componente HP) e uma formação sólida na camada epiderme (componente PE).

(hidradenoma poroide) e da epiderme (poroma écrino). A maioria das neoplasias era da derme. Essa porção era caracterizada por partes císticas e sólidas. A parte sólida era a parede do cisto, abrangendo uma ou duas camadas de células poroides e cuticulares com citoplasma rosa (-Fig. 4). A porção da epiderme era caracterizada por um tumor sólido, consistindo principalmente de células cuboides pequenas, escuras e monomórficas, com citoplasma escasso e núcleo redondo, conhecidas como células poroides (-Fig. 4). Não havia características histológicas de malignidade.

Após 43 meses de acompanhamento, o paciente permanece assintomático e totalmente ativo. Ele voltou às suas atividades anteriores sem limitações físicas. Não foram observados sinais clínicos de recidiva até agora (-Fig. 5).

\section{Discussão}

A coexistência de PE e HP é rara, restrita a poucos relatos de casos no joelho e nas partes superior e inferior das costas. ${ }^{8,9}$ Porém, eles têm demonstrado evolução clínica satisfatória após a excisão completa do tumor. Não foi observada

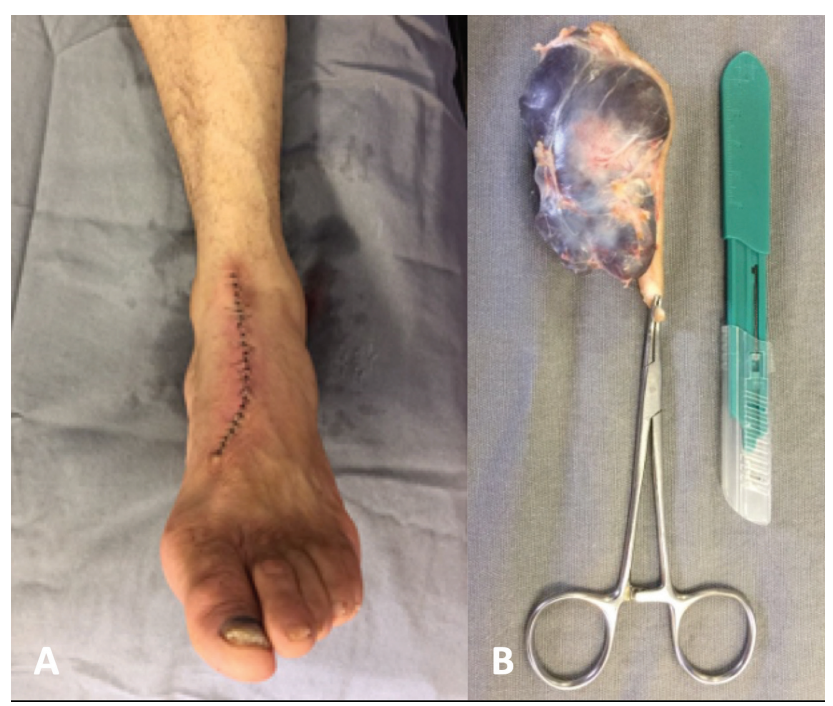

Fig. 3 (A) Abordagem dorsal e (B) tumor totalmente excisado. Após a ressecção, observou-se o aspecto cístico da lesão.

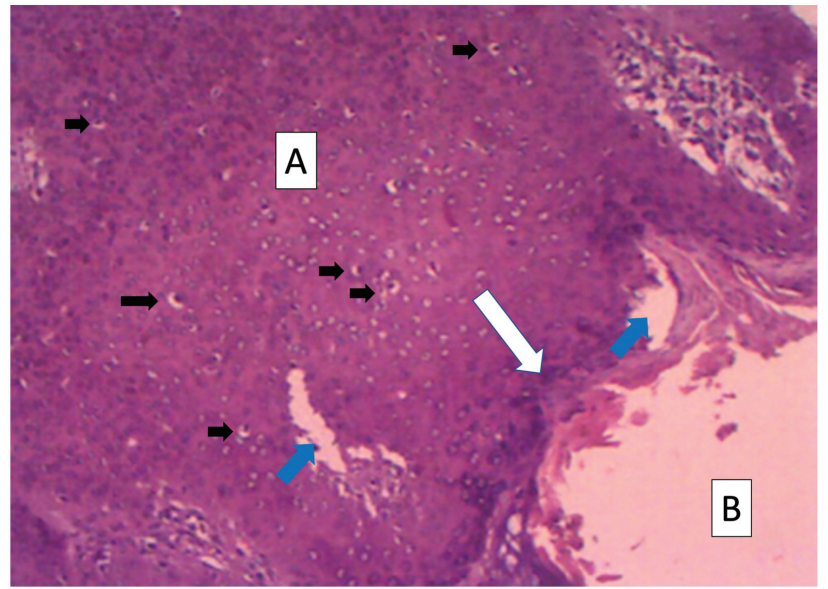

Fig. 4 Exame histopatológico mostrando que a neoplasia era constituída por componentes epidérmicos (poroma écrino) e dérmicos (hidradenoma poroide). O componente marrom escuro (A) corresponde ao componente epiderme da neoplasia, enquanto o componente claro (B) corresponde ao componente derme. O componente da epiderme estava repleto de pequenas células uniformemente cuboides (setas pretas); também foram encontrados alguns espaços ductais relacionados dentro do tumor (setas azuis). O componente da derme apresentou-se como tumor cístico com conteúdo líquido circundado por uma parede sólida (seta branca). A parede do cisto era constituída por camadas de células poroides e cuticulares achatadas. (Hematoxilina-Eosina 100x).

nenhuma característica de malignidade na análise patológica e nenhum sinal de recorrência. O presente estudo relata um caso único de grande tumor solitário sobre o pé, consistindo de uma associação de PE e HP, que foi tratado com sucesso através da ressecção cirúrgica, sem sinais de malignidade e recorrência. Até onde se tem conhecimento, apenas descrições de apresentações separadas desses tumores nos pés foram publicadas.

O PE e o HP compartilham a origem histológica, as características celulares semelhantes às células tumorais monomórficas, a diferenciação ductal e a necrose. ${ }^{7}$ No entanto, clinicamente, apresentam aspectos diferentes. Frequentemente, o PE é uma lesão sólida não cística, com aspectos nodulares, granulares e papilados de várias cores. O HP se apresenta como um tumor sólido e cístico localizado

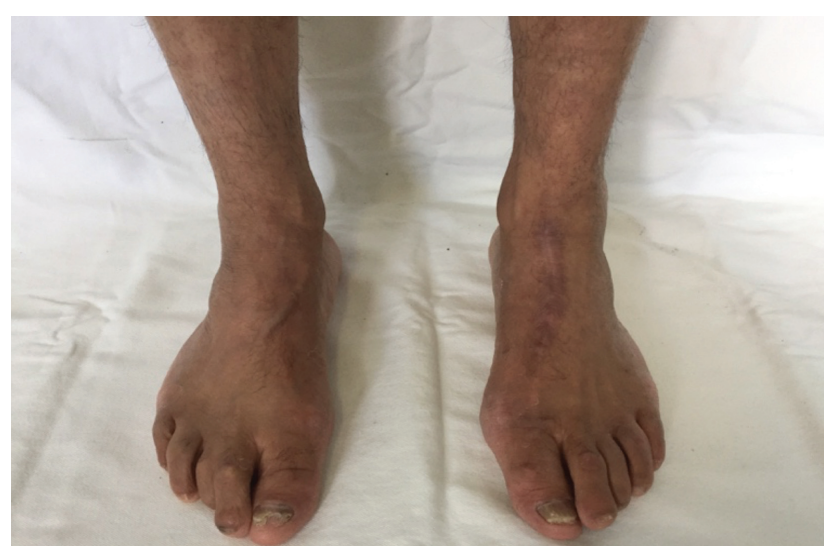

Fig. 5 Aspecto clínico do acompanhamento após 43 meses da excisão do tumor. Não havia sinais de recidiva da massa na região dorsal do pé esquerdo. 
inteiramente na derme. ${ }^{6}$ Diferentemente do PE, o HP não ocorre na palma da mão ou na planta do pé.7 Nosso paciente apresentava uma lesão solitária com características clínicas de PE e HP, nas formas de uma mancha nodular hipercrômica e de uma formação cística, respectivamente.

A etiologia desses tumores é desconhecida. A inflamação e a regeneração tissular podem ter grande importância, já que existem descrições de associação a traumas, cicatrizes, exposição à radiação e imunossupressão. ${ }^{7}$ No entanto, o tumor do nosso paciente cresceu sem qualquer trauma, infecção local anterior, doenças imunossupressoras ou histórico de exposição à radiação. Em geral, uma das hipóteses etiológicas dessa associação é porque, provavelmente, algumas partes da pele e das glândulas sudoríparas podem ser induzidas à via da tumorigênese ao mesmo tempo. ${ }^{5}$ Embora raro, o PE pode evoluir para um porocarcinoma écrino (PCE). $)^{2,10}$

A descrição do diagnóstico e do tratamento desse tumor raro pode ajudar o cirurgião ortopédico na busca por tumores cutâneos como um diagnóstico diferencial de tumores nos pés. Ficou demonstrado que a excisão cirúrgica foi um tratamento eficaz, sem recorrência do tumor no acompanhamento de médio prazo.

\section{Suporte Financeiro}

Não houve suporte financeiro de fontes públicas, comerciais, ou sem fins lucrativos.

\section{Conflito de interesses}

Os autores declaram não ter conflito de interesses.

\section{Referências}

1 Moeller CA, Welch RH, Kaplan DL. An enlarging tumor of the foot. Eccrine poroma. Arch Dermatol 1987;123(05):653-654, 656

2 Wong MW, Tse GM. Eccrine poroma: a differential diagnosis in chronic foot lesions. Foot Ankle Int 2003;24(10):789-792

3 Soyer HP. Neoplasms with eccrine differentiation. Acta Dermatovenerol APA 1997;6(04):151-154

4 Abenoza P, Ackerman AB. Neoplasms With Eccrine Differentiation: Ackerman's Histologic Diagnosis of Neoplastic Skin Diseases: A Method by Pattern Analysis. Philadelphia: Lea \& Febiger; 1990:536

5 Chiu HH, Lan CC, Wu CS, Chen GS, Tsai KB, Chen PH. A single lesion showing features of pigmented eccrine poroma and poroid hidradenoma. J Cutan Pathol 2008;35(09):861-865

6 Misago N, Kohda H. A single lesion demonstrating features of eccrine poroma and poroid hidradenoma. J Dermatol 1995;22 (10):773-779

7 Soyer HP. Neoplasms with eccrine differentiation. Acta Dermatovenerologica 1997;06(97):151-154

8 Casper DJ, Glass LF, Shenefelt PD. An unusually large eccrine poroma: a case report and review of the literature. Cutis 2011; 88(05):227-229

9 Mulinari-Brenner FA, Mukai MM, Bastos CA, Filho EA, Santamaria JR, Neto JF. Porocarcinoma écrino: relato de quatro casos e revisão da literatura [Eccrine porocarcinoma: report of four cases and literature review]. An Bras Dermatol 2009;84(05):519-523

10 Galadari E, Mehregan AH, Lee KC. Malignant transformation of eccrine tumors. J Cutan Pathol 1987;14(01):15-22 